\title{
Bone turnover markers may predict the progression of osteonecrosis of the femoral head in aged males
}

\author{
Zhenyu Shi $^{1,2 \#}$, Hongting Jin ${ }^{1,3 \#}$, Quanwei Ding ${ }^{1,2}$, Jun Ying ${ }^{1,2}$, Ping-Er Wang ${ }^{1}$, Peijian Tong ${ }^{3}$, \\ Bangjian $\mathrm{He}^{3}$
}

${ }^{1}$ Institute of Orthopadics and Traumatology, the First Affiliated Hospital of Zhejiang Chinese Medical University, Hangzhou 310053 , China; ${ }^{2}$ The First College of Clinical Medicine, Zhejiang Chinese Medical University, Hangzhou 310053, China; ${ }^{3}$ Department of Orthopedic Surgery, the First Affiliated Hospital of Zhejiang Chinese Medical University, Hangzhou 310006, China

Contributions: (I) Conception and design: P Tong, B He; (II) Administrative support: P Tong; (III) Provision of study materials or patients: H Jin, P Tong, B He; (IV) Collection and assembly of data: Z Shi, H Jin, Q Ding; (V) Data analysis and interpretation: Z Shi, PE Wang; (VI) Manuscript writing: All authors; (VII) Final approval of manuscript: All authors.

"These authors contributed equally to this work.

Correspondence to: Bangjian He; Peijian Tong. Department of Orthopedic Surgery, the First Affiliated Hospital of Zhejiang Chinese Medical University, No. 54, Youdian Road, Hangzhou 310006, China. Email: hebangjian@163.com; tongpeijian@163.com.

Background: Recent studies suggest that the imbalance of bone metabolism is associated with the pathogenesis of osteonecrosis of the femoral head (ONFH). However, limited data exist on the bone turnover markers (BTMs) in these patients compared with the healthy control (HC) comprehensively.

Methods: In total, 196 participants aged 29-83 [53 patients were excluded, 70 nontraumatic ONFH (mean age 57.75 \pm 12.61 ; mean BMI 24.10 \pm 3.04 ) and $73 \mathrm{HC}$ (mean age 54.04 \pm 11.12 ; mean BMI 23.67 \pm 3.64 )] were recruited in our cross-sectional study. Osteocalcin (OC), t-P1NP (N-terminal procollagen type 1 extension pro-peptide), $\beta$-CTx (beta-isomerized type I collagen C-telopeptide breakdown products), 25-hydroxycholecalciferol, and parathormone (PTH) were measured using automated analyser.

Results: In comparison to controls, nontraumatic ONFH patients have lower 25(OH)D levels and higher levels of $\mathrm{t}-\mathrm{P} 1 \mathrm{NP}, \beta-\mathrm{CTx}$ and $\mathrm{OC}(\mathrm{P}<0.01)$. But there was no significant difference in PTH levels between these two groups. Logistic regression analysis revealed that low 25(OH)D and high t-P1NP were significantly associated with nontraumatic ONFH. ROC curve analysis showed the highest AUC for t-P1NP+25(OH)D model [t-P1NP+25(OH)D: 0.702, 95\% CI: 0.620-0.776; t-P1NP: 0.621, 95\% CI: $0.536-0.701 ; 25(\mathrm{OH}) \mathrm{D}: 0.678,95 \%$ CI: $0.594-0.753]$. With regard to the cutoff values calculated from the ROC curves, t-P1NP+25(OH)D model showed $64.29 \%$ sensitivity and $73.97 \%$ specificity. The deficiency of $25(\mathrm{OH}) \mathrm{D}$ occurred at all phases, and other BTMs were in a high rate during different phases according to the ARCO classification.

Conclusions: Both 25(OH)D deficiency and high t-P1NP were independent risk factors for nontraumatic ONFH. Our results suggest that BTMs, t-P1NP+25(OH)D model (t-P1NP $\geq 54.82 \mathrm{ng} / \mathrm{mL}$ and $25(\mathrm{OH}) \mathrm{D}$ $\leq 21.86 \mathrm{ng} / \mathrm{mL}$ ), may facilitate to diagnose nontraumatic ONFH in aged male patients.

Keywords: Nontraumatic osteonecrosis of the femoral head (nontraumatic ONFH); bone turnover markers (BTMs); progression; male

Submitted Jul 09, 2019. Accepted for publication Oct 17, 2019.

doi: 10.21037/atm.2019.11.22

View this article at: http://dx.doi.org/10.21037/atm.2019.11.22 


\section{Introduction}

Nontraumatic osteonecrosis of the femoral head (ONFH), a multi-factorial devastating joint disease with unclear pathological mechanism, has been highly associated with high-dose or chronic corticosteroid use, alcohol abuse, sickle cell disease, HIV, as well as lupus (1-3). This increasing worldwide health problem, unfortunately, is progressing spontaneously, necessitating various preserving procedures and most culminating in total hip arthroplasty (4).

Early diagnosis is of upmost significance. Multiple studies have indicated that the repair reaction, especially excessive bone resorption in bone remodeling, results in the collapse progression (5-8). Bone turnover markers (BTMs) can be used to monitor the rate between bone formation and resorption. But there is no report concerning BTMs in nontraumatic $\mathrm{ONFH}$ patients compared with that of healthy control (HC) actually.

Reviewing literature the following five BTMs were found as possible predicting parameters for nontraumatic ONFH: osteocalcin, t-P1NP (N-terminal procollagen type 1 extension pro-peptide), $\beta$-CTx (beta-isomerized type I collagen C-telopeptide breakdown products), 25-hydroxy-cholecalciferol, and parathormone (PTH) $(9,10)$.

We hypothesized that BTMs show differences in patients. Therefore, in the current study, we investigated the characteristics of BTM levels for nontraumatic ONFH in comparison with healthy groups.

\section{Methods}

\section{Study design and participants}

This cross-sectional study is a sub-cohort analysis of a study on BTM levels conducted in Health Examination Center and Department of Orthopedic \& Traumatology, the First Affiliated Hospital of Zhejiang Chinese Medical University in Hangzhou, from July 2017 to December 2018, using a total of 196 participants aged 29-83 (data on file). For the purpose of this study, patients with one of the following conditions were excluded: the diagnosis of nontraumatic ONFH was not confirmed by radiographs and MRI; renal insufficiency; metabolic bone diseases, such as osteomalacia or Paget's disease of the bone; secondary causes of osteoporosis, such as Cushing's disease, hyperthyroidism, Crohn's disease, or rheumatoid arthritis; use of oral bisphosphonates, parathyroid hormone, strontium ranelate, calcitonin, or any other investigational therapy within half a year. The presented study was approved by the Ethical Committee of the First Affiliated Hospital of Zhejiang Chinese Medical University (Ethical approval ID: 2018KL-005).

\section{Sample collection and BTM analyses}

Serum samples were collected under fasting conditions in all participants. All BTMs were performed at Clinical Laboratory using a COBAS e 601 automated analyser (Roche Diagnostics, Germany) by ECLIA according to the manufacturer's instructions. The intra- and inter-assay CVs are $<10 \%$ and $<15 \%$ respectively. The t-P1NP (Elecsys ${ }^{\circledR}$ total $\mathrm{P} 1 \mathrm{NP}$ ) repeatability and intermediate precision were reported as $<3.3 \%$ and $<3.8 \%$ respectively, with a lower limit of quantification (LLOQ) and upper limit of quantification (ULOQ) of 5 and $1,200 \mathrm{ng} / \mathrm{mL}$ respectively. The $\beta$-CTx (Elecsys ${ }^{\circledR} \beta$-CrossLaps/serum) repeatability and intermediate precision were reported as $<4.8 \%$ and $<5.8 \%$ respectively, with an LLOQ and ULOQ of 0.010 and $6.00 \mathrm{ng} / \mathrm{mL}$ respectively. The PTH (Elecsys ${ }^{\circledR}$ PTH) repeatability and intermediate precision were reported as $<2.1 \%$ and $<3.5 \%$ respectively, with an LLOQ and ULOQ of 1.20 and 5,000 $\mathrm{pg} / \mathrm{mL}$ respectively. The $25(\mathrm{OH}) \mathrm{D}$ (Elecsys ${ }^{\circledR}$ Vitamin D total) repeatability and intermediate precision were reported as $<6.9 \%$ and $<13.2 \%$ respectively, with an LLOQ and ULOQ of 3.00 and $70.0 \mathrm{ng} / \mathrm{mL}$ respectively. The OC (Elecsys ${ }^{\circledR} \mathrm{N}-\mathrm{MID}$ Osteocalcin) repeatability and intermediate precision were reported as $<1.4 \%$ and $<2.4 \%$ respectively, with an LLOQ and ULOQ of 0.500 and $300 \mathrm{ng} / \mathrm{mL}$ respectively.

\section{Statistical analysis}

Independent $t$ test and the chi-square test was used to compare the baseline and BTM date of the two groups. Logistic analysis was conducted to determine the associations between abnormally BTMs in nontraumatic ONFH. Receiver operating characteristic (ROC) curves were plotted for obtaining serum BTM cutoff values for nontraumatic ONFH diagnosis. The area under the curve (AUC) was calculated, and the maximum sensitivity and specificity was determined by the cutoff value. All tests were two-sided, and $\mathrm{P}<0.05$ was considered to be significant. The statistical software used was the Statistical Package for Social Sciences (SPSS) version 22 (IBM Corp., NY, USA). 


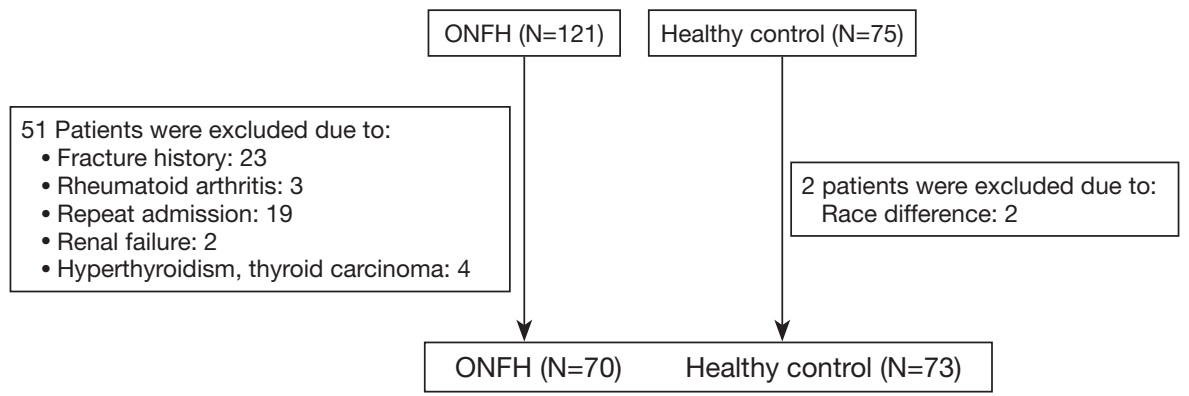

Figure 1 This is a flow diagram illustrating patient inclusion. ONFH, osteonecrosis of the femoral head.

Table 1 Clinical characteristics of participants

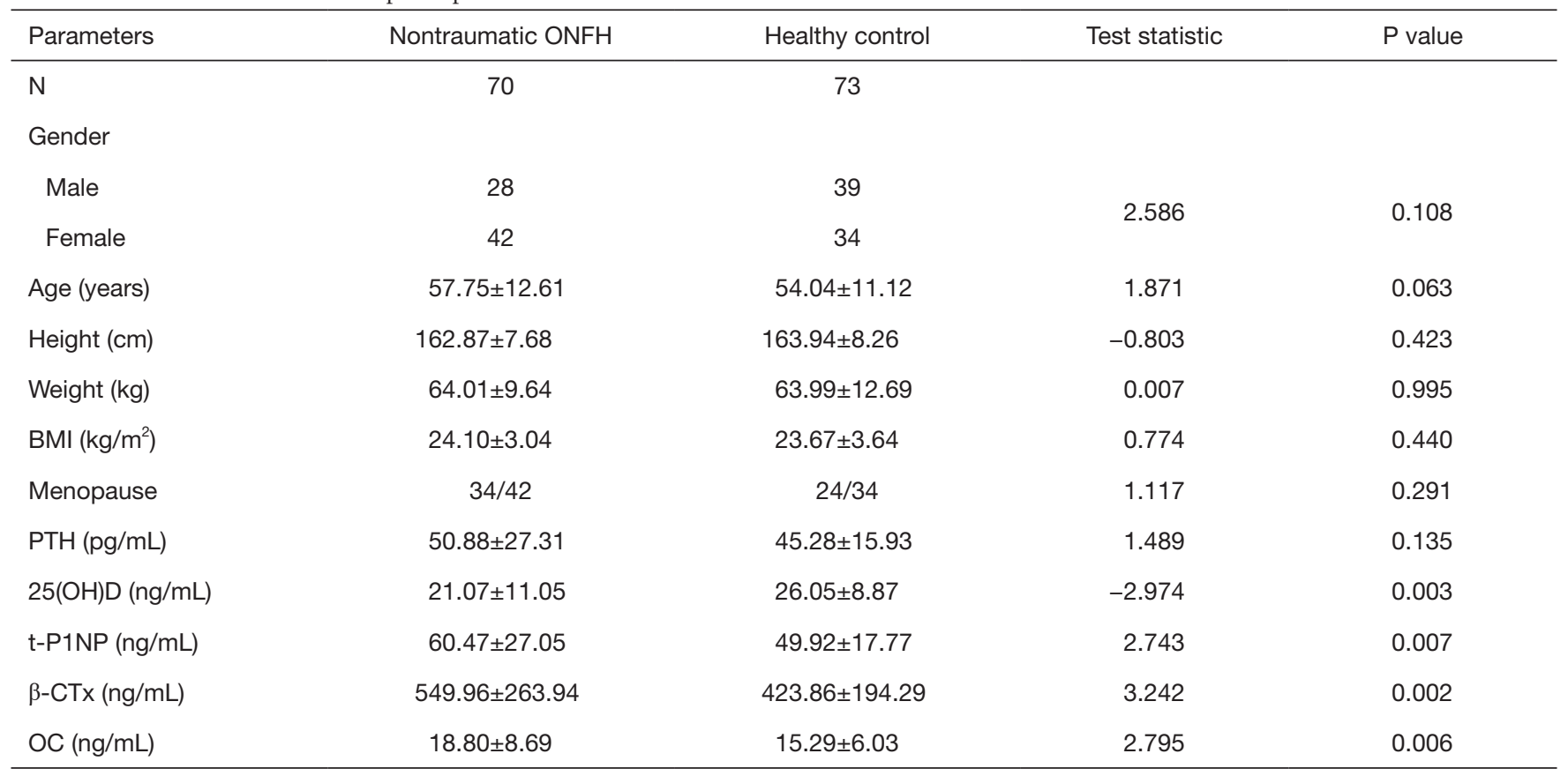

ONFH, osteonecrosis of the femoral head; PTH, parathormone; OC, osteocalcin.

\section{Results}

Verified the 196 selected participants, 51 patients (23 cases have fracture history; 3 cases were diagnosed as rheumatoid arthritis; 19 cases duplicated due to the repeat admission; 2 cases were diagnosed as renal failure; 4 cases were diagnosed as hyperthyroidism or thyroid carcinoma) and 2 cases ( 2 cases because of race difference) were excluded (Figure 1). The baseline demographics were similar between the nontraumatic ONFH and the $\mathrm{HC}$ (Table 1).

25(OH)D levels were significantly higher in the $\mathrm{HC}$ than in the nontraumatic ONFH patients $(\mathrm{P}<0.01)$ patients (nontraumatic ONFH: $21.07 \pm 11.05 \mathrm{ng} / \mathrm{mL}$; HC: $26.05 \pm 8.87 \mathrm{ng} / \mathrm{mL}$ ) (Table 1). t-P1NP levels were signif icantly higher in the nontraumatic ONFH patients

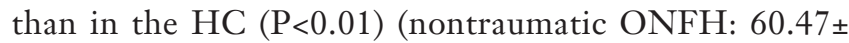
$27.05 \mathrm{ng} / \mathrm{mL}$; HC: $49.92 \pm 17.77 \mathrm{ng} / \mathrm{mL})$. $\beta$-CTx levels were significantly higher in the nontraumatic $\mathrm{ONFH}$ patients than in the $\mathrm{HC}(\mathrm{P}<0.01)$ (nontraumatic $\mathrm{ONFH}$ : $549.96 \pm 263.94 \mathrm{ng} / \mathrm{mL}$; HC: $423.86 \pm 194.29 \mathrm{ng} / \mathrm{mL})$. OC levels were significantly higher in the nontraumatic $\mathrm{ONFH}$ patients than in the $\mathrm{HC}(\mathrm{P}<0.01)$ (nontraumatic ONFH: $18.80 \pm 8.69 \mathrm{ng} / \mathrm{mL}$; HC: $15.29 \pm 6.03 \mathrm{ng} / \mathrm{mL})$. There was no significant difference in PTH levels between this two groups. 
Table 2 Logistic regression analysis odds ratio of low 25(OH)D and high t-P1NP

\begin{tabular}{lcccccr}
\hline & B & S.E. & Wald & P value & OR & 95\% Cl \\
\hline Intercept & -0.784 & 0.241 & 10.558 & 0.01 & 0.458 & \\
25(OH)D & 1.525 & 0.372 & 16.766 & 0.00 & 4.595 & $2.214-9.534$ \\
t-P1NP & 2.238 & 1.105 & 4.101 & 0.043 & 9.372 & $1.074-81.741$ \\
\hline
\end{tabular}

OR, odds ratio; $\mathrm{Cl}$, confidence interval.
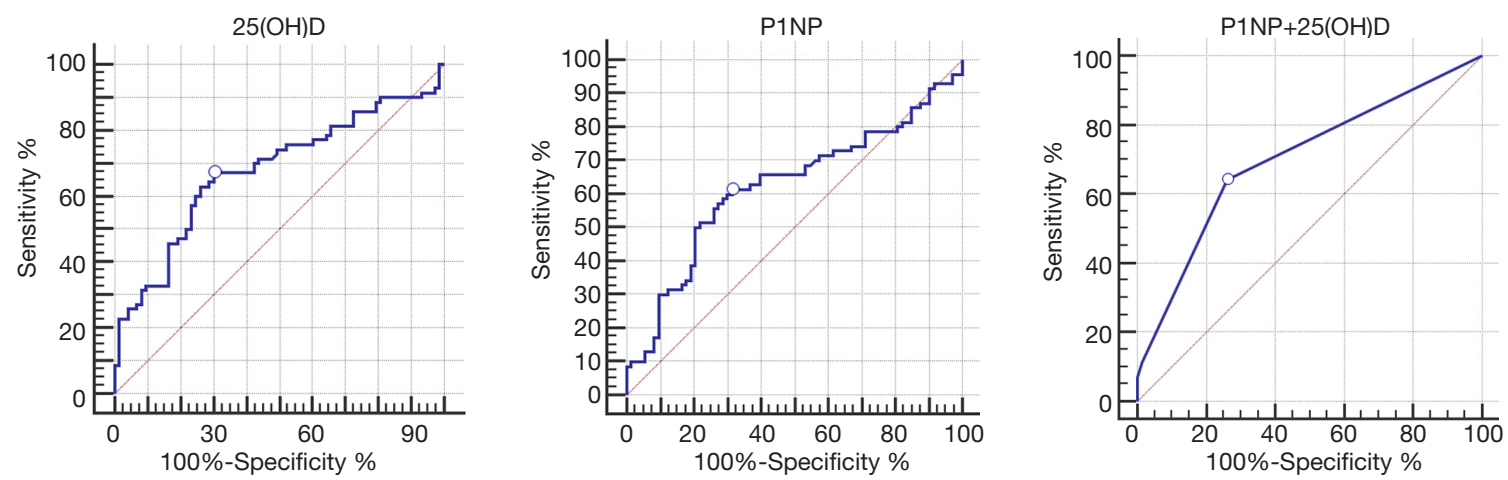

Figure 2 Receiver operating characteristic (ROC) curve analysis of 25(OH)D, P1NP and P1NP+25(OH)D model for diagnosis of nontraumatic ONFH compared with healthy control. The area under the curve (AUC) values were 25(OH)D: 0.678; P1NP: 0.621; and P1NP+25(OH)D: 0.702 .

The value in the reference limitation is defined as 0 , and the value outside as 1 . To our surprise, all abnormalities were unidirectional compared to the HC. After adjustment for confounding factors, logistic regression analysis revealed low $25(\mathrm{OH}) \mathrm{D}$ and high $\mathrm{t}-\mathrm{P} 1 \mathrm{NP}$ were significantly associated with nontraumatic ONFH (Table 2): low 25(OH) $\mathrm{D}$ with odds ratio (OR) of 4.595 (95\% CI: 2.214-9.534, $\mathrm{P}<0.01$ ), high t-P1NP with OR of 9.372 (95\% CI: 1.074 $81.741, \mathrm{P}<0.05$ ) when compared with that of the HC group.

For distinguishing nontraumatic ONFH from the HC group, ROC curve analysis showed the highest AUC for t-P1NP+25(OH)D model [t-P1NP+25(OH)D: 0.702, 95\% CI: 0.620-0.776; t-P1NP: 0.621, 95\% CI: 0.536-0.701; 25(OH) D: $0.678,95 \%$ CI: $0.594-0.753]$. With regard to the cutoff values calculated from the ROC curves, $t-\mathrm{P} 1 \mathrm{NP}+25(\mathrm{OH}) \mathrm{D}$ model showed $64.29 \%$ sensitivity and $73.97 \%$ specificity (cutoff, $0.313)$, t-P1NP levels showed $61.43 \%$ sensitivity and $68.49 \%$ specificity (cutoff, $54.82 \mathrm{ng} / \mathrm{mL}$ ), 25(OH)D levels showed $67.14 \%$ sensitivity and $69.86 \%$ specificity (cutoff, $21.86 \mathrm{ng} / \mathrm{mL}$ ) (Figure 2).

Lastly, the changes of BTMs during different phases were shown in Figure 3 according to the ARCO classification. Detailed descriptions of BTMs among different phases were shown in Table 3. Due to the difference in sample size, comparisons of BTMs were among particular phases. The deficiency of $25(\mathrm{OH}) \mathrm{D}$ becomes more obvious as the disease progresses. $\mathrm{t}-\mathrm{P} 1 \mathrm{NP}$ levels, $\beta$-CTx levels and OC levels show nearly the same trend during different phases.

\section{Discussion}

In this study, we have tried to find out the characteristics of BTM levels for nontraumatic ONFH compared with the health control. t-P1NP, OC and $\beta$-CTx levels were significantly higher in the nontraumatic ONFH patients, instead of $25(\mathrm{OH}) \mathrm{D}$ being lower. Bone metabolic disturbance, high level bone formation and resorption, is what we can directly observe. The anomalies of indicators are similar both in nontraumatic ONFH and the health control (PTH, t-P1NP, $\beta$-CTx and OC showed an abnormal elevation, while $25(\mathrm{OH}) \mathrm{D}$ displayed a decrease).

Many studies have reported the alendronate treatment, a bisphosphonate, could delay the collapse in nontraumatic ONFH with pre-collapse stags by inhibiting the activity of the osteoclasts $(8,11)$. According to the results of data analysis, $\beta$-CTx levels were higher in the nontraumatic 

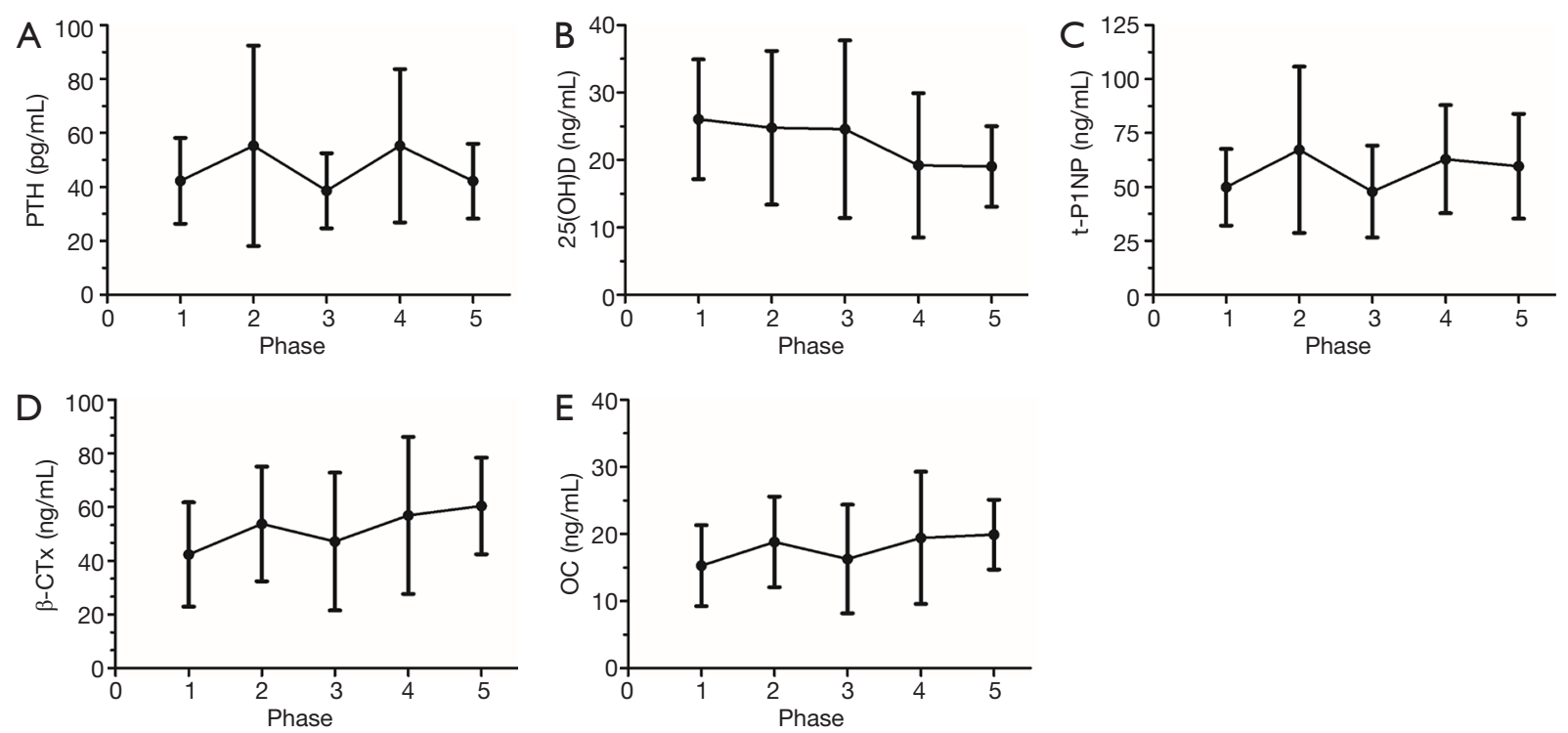

Figure 3 The changes of BTMs during different phases. Results of PTH (parathormone, A), 25(OH)D (25-hydroxy-cholecalciferol, B), t-P1NP (N-terminal procollagen type 1 extension pro-peptide, C), $\beta$-CTx (beta-isomerized type I collagen C-telopeptide breakdown products, D) and OC (osteocalcin, E) are indicated as mean + standard deviation. BTM, bone turnover markers; OC, osteocalcin.

Table 3 Comparisons of bone turnover markers at different phases

\begin{tabular}{|c|c|c|c|c|c|}
\hline Parameters & Phase I & Phase II & Phase III & Phase IV & Phase V \\
\hline Age (years) & $54.04 \pm 11.12$ & $59.00 \pm 10.20$ & $53.61 \pm 12.50$ & $59.00 \pm 13.22$ & $56.57 \pm 13.53$ \\
\hline Height $(\mathrm{cm})$ & $163.94 \pm 8.26$ & $165.40 \pm 7.66$ & $165.11 \pm 6.03$ & $161.70 \pm 8.44$ & $161.21 \pm 4.74$ \\
\hline Weight $(\mathrm{kg})$ & $63.99 \pm 12.69$ & $67.50 \pm 8.68$ & $64.36 \pm 9.70$ & $63.07 \pm 9.72$ & $63.07 \pm 11.23$ \\
\hline PTH (pg/mL) & $45.28 \pm 15.93$ & $55.32 \pm 37.16$ & $38.59 \pm 13.92$ & $55.28 \pm 28.43$ & $42.18 \pm 13.89$ \\
\hline 25(OH)D (ng/mL) & $26.05 \pm 8.87$ & $24.80 \pm 11.40$ & $24.58 \pm 13.16$ & $19.22 \pm 10.71$ & $19.04 \pm 5.97$ \\
\hline t-P1NP (ng/mL) & $49.92 \pm 17.77$ & $67.21 \pm 38.56$ & $47.91 \pm 21.33$ & $62.90 \pm 25.05$ & $59.64 \pm 24.24$ \\
\hline$\beta-\mathrm{CT} x(\mathrm{ng} / \mathrm{mL})$ & $423.86 \pm 194.29$ & $537.58 \pm 213.57$ & $472.18 \pm 256.76$ & $569.54 \pm 292.38$ & $604.75 \pm 180.18$ \\
\hline
\end{tabular}

ONFH, osteonecrosis of the femoral head; PTH, parathormone; OC, osteocalcin.

ONFH patients. There was no statistical difference in the number of abnormal increases. We hold the view that bone resorption should not be the only concern in nonsurgical treatment, the bone homeostasis, dynamic balance of bone resorption and bone formation, may be the best result. Logistic regression analysis revealed low 25(OH) $\mathrm{D}$ and high t-P1NP were significantly associated with nontraumatic ONFH. t-P1NP+25(OH)D model (t-P1NP $\geq 54.82 \mathrm{ng} / \mathrm{mL}$ and $25(\mathrm{OH}) \mathrm{D} \leq 21.86 \mathrm{ng} / \mathrm{mL})$ may be specific for nontraumatic ONFH based on ROC curves. Stage IV is a separate stage for lesions, sclerosis, associated with flattening of the femoral head (12). Abnormally high t-P1NP may explain this collapse progression. Five of the six abnormal t-P1NP patients were aged male. This may show the diagnostic significance of the model for aged male patients. 
This study selected OC and t-P1NP to monitor the bone formation, $\beta$-CTx is chosen as bone resorption marker $(13,14)$. $25(\mathrm{OH}) \mathrm{D}$ plays a fundamental role in maintaining human skeleton (15). And the role of PTH cannot be underemphasized, it regulates serum calcium through effects on bone, kidney and intestine $(12,16)$.

In order to confirm that the results we found were not isolated phenomena, we set the health control as the first stage and plotted the currently collected data into a line graph to show the changing trend. The deficiency of $25(\mathrm{OH}) \mathrm{D}$ occurred at all phases, and its severity increases as the disease progresses. $\mathrm{t}-\mathrm{P} 1 \mathrm{NP}$ and $\beta-\mathrm{CT}$ x showed similar trend. The process of ONFH matches the mechanism of microfracture repairation, a high rate of the bone turnover process (17). It also raises questions about whether drugs that inhibit osteoclasts are appropriate in nontraumatic ONFH patients and the dynamic balance should be broken or not.

The present study had some limitations. First, this study was designed to avoid the effects of operations and drugs, while the leaving small sample size and the regionalization of the population may affect the results in particular. Further studies on larger populations should be investigated. Also, confounding variables such as nutritional habits, housework-related physical activity, which may affect circulating BTMs potentially, should be taken into consideration. The physical condition of the control group may require further analysis and screening. Lastly, most of the included cases were inpatients, different disease stages and causes are impossible to further compare because of the large sample size gap.

Despite several limitations, this is the first study to determine associations between BTMs with the diagnosis of nontraumatic ONFH compared with the HC and five common bone turnover parameters were all tested. Furthermore, the modeling results by ROC curves also provide references for early diagnosis. And, there existing a problem that large standard deviations were found in the statistics of BTMs trends, but it does not affect the result judgment for abnormal bone metabolism.

In conclusion, we found that both $25(\mathrm{OH}) \mathrm{D}$ deficiency and high t-P1NP were independent risk factors for nontraumatic ONFH. BTMs, t-P1NP+25(OH)D model $(\mathrm{t}-\mathrm{P} 1 \mathrm{NP} \geq 54.82 \mathrm{ng} / \mathrm{mL}$ and $25(\mathrm{OH}) \mathrm{D} \leq 21.86 \mathrm{ng} / \mathrm{mL}$ ), may help to diagnose nontraumatic ONFH for aged male patients. High rate of the bone turnover process is one of the pathological mechanisms for nontraumatic ONFH, and the kinetic of BTMs is helpful to choose the best treatment in different phase. Abnormal bone formation also needs more attention on the diagnosis and treatment of nontraumatic ONFH.

\section{Acknowledgments}

None.

\section{Footnote}

Conflicts of Interest: The authors have no conflicts of interest to declare.

Ethical Statement: The authors are accountable for all aspects of the work in ensuring that questions related to the accuracy or integrity of any part of the work are appropriately investigated and resolved. The presented study was approved by the Ethical Committee of the First Affiliated Hospital of Zhejiang Chinese Medical University (Ethical approval ID: 2018-KL-005).

\section{References}

1. Mont MA, Etienne G, Ragland PS. Outcome of nonvascularized bone grafting for osteonecrosis of the femoral head. Clin Orthop Relat Res 2003;(417):84-92.

2. Hernigou P, Trousselier M, Roubineau F, et al. Stem Cell Therapy for the Treatment of Hip Osteonecrosis: A 30Year Review of Progress. Clin Orthop Surg 2016;8:1-8.

3. Larson E, Jones LC, Goodman SB, et al. Early-stage osteonecrosis of the femoral head: where are we and where are we going in year 2018? Int Orthop 2018;42:1723-8.

4. Mont MA, Cherian JJ, Sierra RJ, et al. Nontraumatic Osteonecrosis of the Femoral Head: Where Do We Stand Today? A Ten-Year Update. J Bone Joint Surg Am 2015;97:1604-27.

5. Agarwala S, Shah S, Joshi VR. The use of alendronate in the treatment of avascular necrosis of the femoral head: follow-up to eight years. J Bone Joint Surg Br 2009;91:1013-8.

6. Li W, Sakai T, Nishii T, et al. Distribution of TRAPpositive cells and expression of HIF-1alpha, VEGF, and FGF-2 in the reparative reaction in patients with osteonecrosis of the femoral head. J Orthop Res 2009;27:694-700.

7. Pierce TP, Elmallah RK, Jauregui JJ, et al. A current review of non-vascularized bone grafting in osteonecrosis of the femoral head. Curr Rev Musculoskelet Med 
$2015 ; 8: 240-5$.

8. Nishii T, Sugano N, Miki H, et al. Does alendronate prevent collapse in osteonecrosis of the femoral head? Clin Orthop Relat Res 2006;443:273-9.

9. Floerkemeier T, Hirsch S, Budde S, et al. Bone turnover markers failed to predict the occurrence of osteonecrosis of the femoral head: a preliminary study. J Clin Lab Anal 2012;26:55-60.

10. Szulc P, Naylor K, Hoyle NR, et al. Use of CTX-I and PINP as bone turnover markers: National Bone Health Alliance recommendations to standardize sample handling and patient preparation to reduce pre-analytical variability. Osteoporos Int 2017;28:2541-56.

11. Lai KA, Shen WJ, Yang CY, et al. The use of alendronate to prevent early collapse of the femoral head in patients with nontraumatic osteonecrosis. A randomized clinical study. J Bone Joint Surg Am 2005;87:2155-9.

12. Sultan AA, Mohamed N, Samuel LT, et al. Classification systems of hip osteonecrosis: an updated review. Int Orthop 2019;43:1089-95.

Cite this article as: Shi Z, Jin H, Ding Q, Ying J, Wang PE, Tong $\mathrm{P}, \mathrm{He} \mathrm{B}$. Bone turnover markers may predict the progression of osteonecrosis of the femoral head in aged males. Ann Transl Med 2019;7(22):626. doi: 10.21037/atm.2019.11.22
13. Nishizawa Y, Ohta H, Miura M, et al. Guidelines for the use of bone metabolic markers in the diagnosis and treatment of osteoporosis (2012 edition). J Bone Miner Metab 2013;31:1-15.

14. Zhang J, Zhang T, Xu X, et al. Zoledronic acid combined with percutaneous kyphoplasty in the treatment of osteoporotic compression fracture in a single T12 or L1 vertebral body in postmenopausal women. Osteoporos Int 2019;30:1475-80.

15. Kubiak RW, Zelnick LR, Hoofnagle AN, et al. Mineral Metabolism Disturbances and Arteriovenous Fistula Maturation. Eur J Vasc Endovasc Surg 2019;57:719-28.

16. Cailleaux PE, Biau D, Leclerc P, et al. Biological secondary contributors to osteoporosis in fractured patients, is an early systematic assay relevant? Joint Bone Spine 2019;86:777-81.

17. Pan C, Liu X, Li T, et al. Kinetic of bone turnover markers after osteoporotic vertebral compression fractures in postmenopausal female. J Orthop Surg Res $2018 ; 13: 314$. 